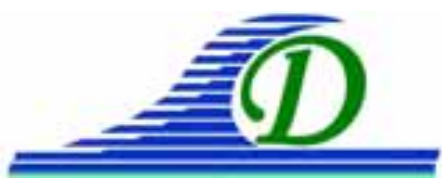
XIII ${ }^{\text {èmes }}$ Journées Nationales Génie Côtier - Génie Civil Dunkerque, 2-4 juillet 2014

DOI:10.5150/jngcgc.2014.037 @ Editions Paralia CFL

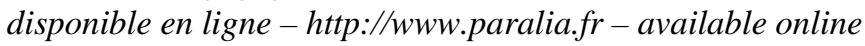

\title{
Etude de stabilité d'une île de sable protégée par des géosynthétiques (GSC) en Guinée équatoriale (Corisco)
}

\author{
Pierre FARNOLE ${ }^{1}$, Julie LEBUNETEL ${ }^{1}$, Brahim EL BOUNI ${ }^{2}$
}

1. ERAMM, 1503 rte des Dolines, BP 42, 06901 Sophia Antipolis, France. jl@eramm.fr ; pf@eramm.fr

2. SOMAGEC GE, Edificio Ureca, Calle Parque Africa, BP 405, Malabo,

République de Guinée équatoriale.brahim.elbouni@somagecge.net

\section{Résumé :}

Le gouvernement guinéen (Guinée équatoriale) a entrepris de développer ces infrastructures et en particulier au sein de ses nombreuses îles dans le golfe de Guinée. Dans ce contexte, l'île de Corisco, située à une vingtaine de kilomètres du continent, fait l'objet de la création d'un port, non pas sur l'île elle-même, mais à une certaine distance de celle-ci pour ne pas impacter l'environnement et la stabilité des plages de sable qui entourent l'île. Le choix a été fait de créer un îlot de sable servant à abriter un port et de relier cet îlot à l'île par un wharf. Notre étude a consisté à dimensionner un ouvrage de protection sous la forme d'empilements de GSC remplis de sable actuellement en cours de construction. La forme et la position de l'îlot a été déterminé en fonction des contraintes hydrodynamiques et sédimentologiques pour un moindre impact. Cette publication a pour objet de mettre en évidence qu'il est parfaitement envisageable de réaliser des digues de protection en géotextile à condition de prendre des précautions dans la conception, le dimensionnement et la construction de l'ouvrage.

Mots-clés : Guinée équatoriale, Port, Ile, Sable, Géosynthétique (Geotextile Sand Containers), Houle, Digue, Dimensionnement.

\section{Introduction}

L'île de Corisco est située à environ $29 \mathrm{~km}$ au Sud-ouest du Rio Muni (estuaire de Kogo, Guinée équatoriale - voir figure 1). Sa superficie est de $14 \mathrm{~km}^{2}$ et son point le plus haut est à $35 \mathrm{~m}$ au-dessus du niveau de la mer. Cette île est directement soumise aux influences du Rio Muni qui charrie de grandes quantités d'alluvions lors de la saison des pluies. Elle est caractérisée par un cordon littoral de sable blanc (200-300 $\mu \mathrm{m})$ qui couvre l'ensemble du littoral et se termine par "une queue de Comète" exactement au Sud-Est de l'île, là où les vagues océaniques d'Ouest font la jonction après avoir contourné l'île par le Nord et par le Sud. Le projet consiste à construire un îlot de sable en forme de demi-lune dont la partie centrale est un plan d'eau abrité pour un usage portuaire. Les digues de protection sont constituées par des tubes empilés depuis le fond jusqu'à un niveau suffisamment haut pour être protégé du risque de submersion. Les tubes sont posés sur un tapis anti-affouillement préalablement ancré 
sur le fond. Notre contribution au projet est de fournir les éléments de dimensionnement de l'ouvrage au regard de sa stabilité, de la protection contre l'agitation et du risque de submersion.
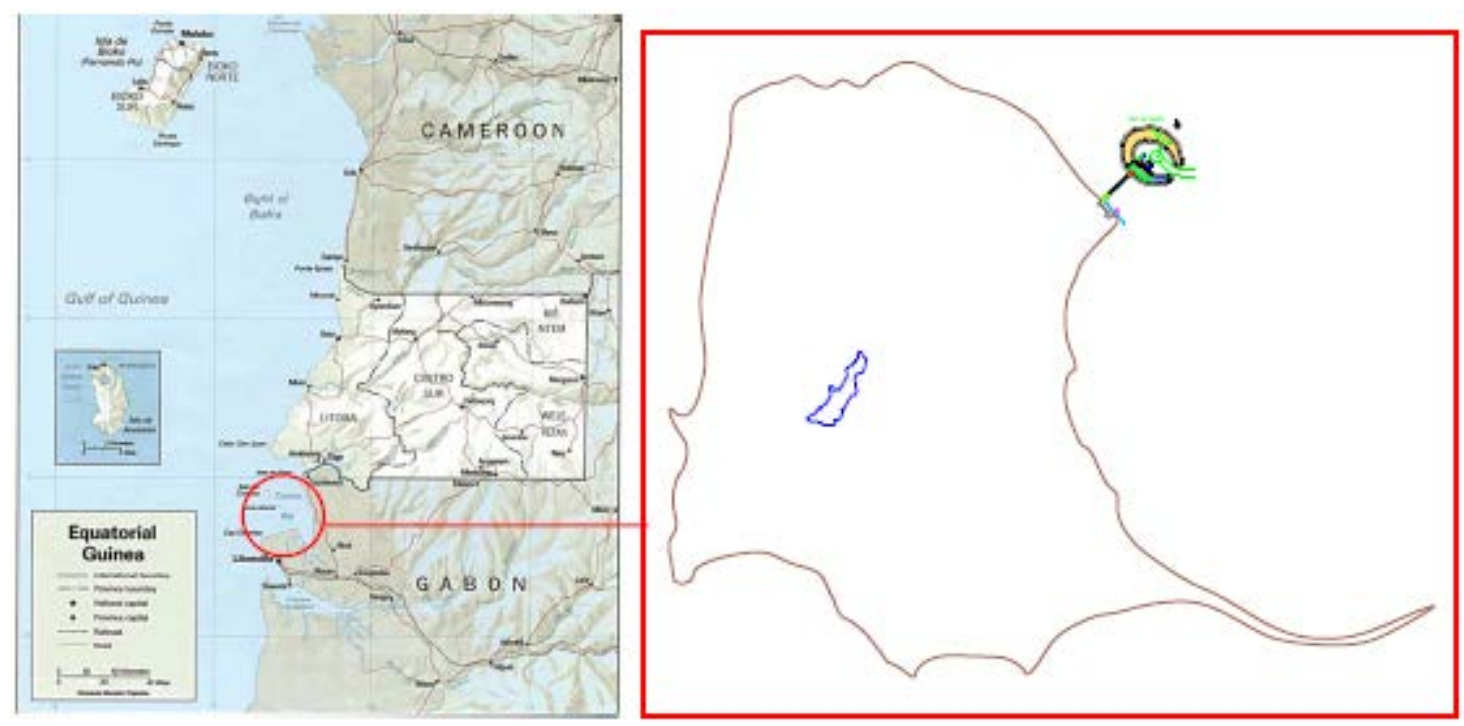

Figure 1. Localisation de l'île de Corisco et du projet.

\section{Les conditions de houle et d'agitation}

Elles sont présentées sur les figures 2, 3, 4 et 5. On note un amortissement des houles du large de secteur SW compris entre $75 \%$ et $80 \%$ après rotation des houles du large de $115^{\circ}$ avant d'atteindre le site de projet. Les caractéristiques de la houle incidente pour différentes périodes de retour sont reportées dans un tableau synthétique. Les valeurs maximales atteignent 1,1 m pour une période de retour cinquantennale. S'agissant de l'agitation générée par les mers de vents de SE dans le plan d'eau intérieur, elle peut atteindre $1 \mathrm{~m}$ en condition annuelle.

Tableau 1. Estimation des hauteurs de houle résiduelles.

\begin{tabular}{|c|c|c|c|c|c|c|c|c|c|c|c|c|}
\hline \multirow{2}{*}{$\begin{array}{l}\operatorname{Tr} \\
\text { (ans) }\end{array}$} & \multirow{2}{*}{$\begin{array}{l}\operatorname{Dir} \\
\left({ }^{\circ} N\right)\end{array}$} & \multirow{2}{*}{$\begin{array}{l}T p \\
(s)\end{array}$} & \multirow{2}{*}{$\begin{array}{l}H_{1 / 10} \\
(m)\end{array}$} & \multirow{2}{*}{$\begin{array}{l}H_{s} \\
(m)\end{array}$} & \multirow{2}{*}{$\begin{array}{l}\text { ho } \\
\text { (mCM) }\end{array}$} & \multicolumn{7}{|c|}{$\begin{array}{l}\text { Estimation des hauteurs de houle } \\
\text { résiduelles }(\mathrm{m})\end{array}$} \\
\hline & & & & & & $P 1$ & P2 & P3 & P4 & P5 & P6 & P7 \\
\hline 1 & 220 & 12 & 3,3 & 2.6 & 2.00 & 0.5 & 0.6 & 0.6 & 0.6 & 0.5 & 0.5 & 0.5 \\
\hline 10 & 220 & 14 & 3,9 & 3,1 & 2.00 & 0.7 & 0.7 & 0.7 & 0.7 & 0.7 & 0.6 & 0.6 \\
\hline 20 & 220 & 14 & 4,2 & 3,3 & 2.20 & 0.9 & 1.0 & 1.0 & 1.0 & 0.9 & 0.9 & 0.9 \\
\hline 50 & 220 & 13 & 4.4 & 3.46 & 2.25 & 1.0 & 1.1 & 1.0 & 1.0 & 1.0 & 0.9 & 0.9 \\
\hline 100 & 220 & 15 & 4.6 & 3,6 & 2.35 & 1.1 & 1.1 & 1.1 & 1.1 & 1.0 & 1.0 & 1.0 \\
\hline
\end{tabular}




\section{XIII ${ }^{\text {èmes }}$ Journées Nationales Génie Côtier - Génie Civil \\ Dunkerque, 2-4 juillet 2014}

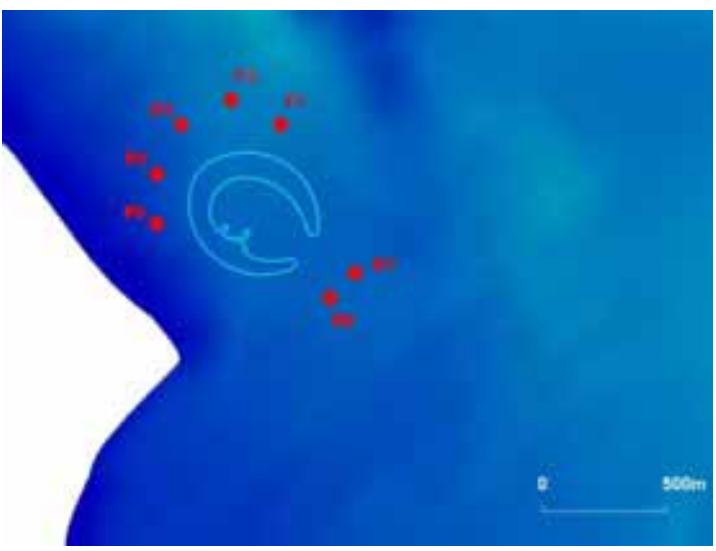

Figure 2. Points de référence de la houle.

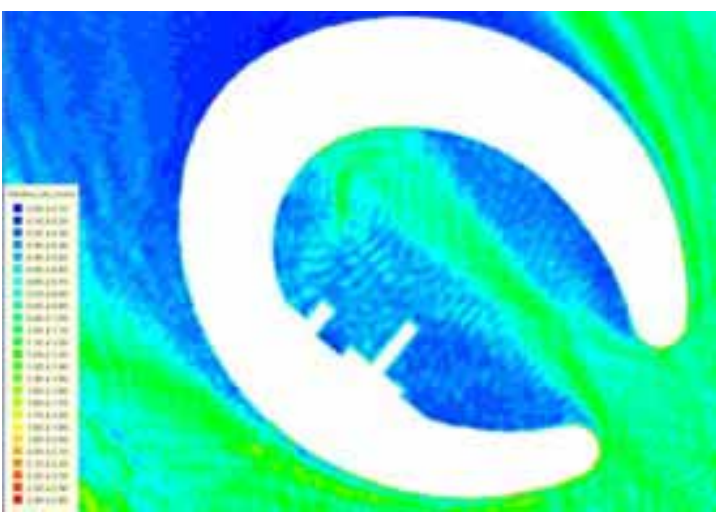

Figure 4. Projet - houle annuelle de SE

$$
\text { Tp : } 4 \text { s. }
$$

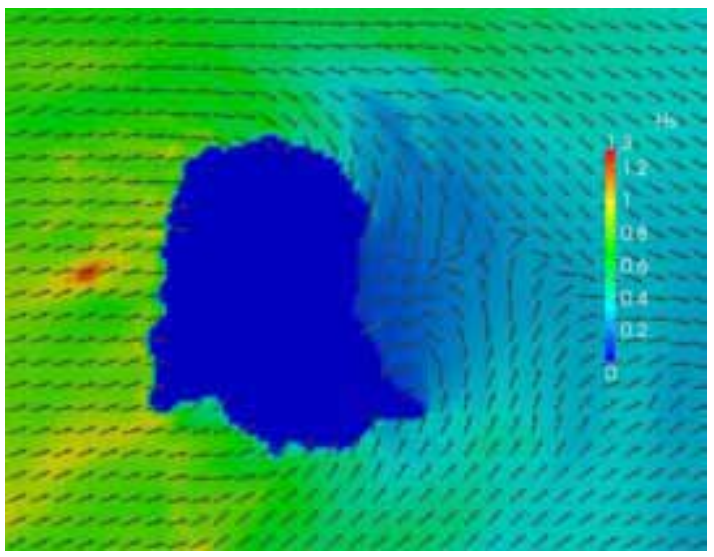

Figure 3. Houle décennale $\left(220^{\circ} \mathrm{N}\right.$, $\mathrm{Tp}=13 \mathrm{~s})$.

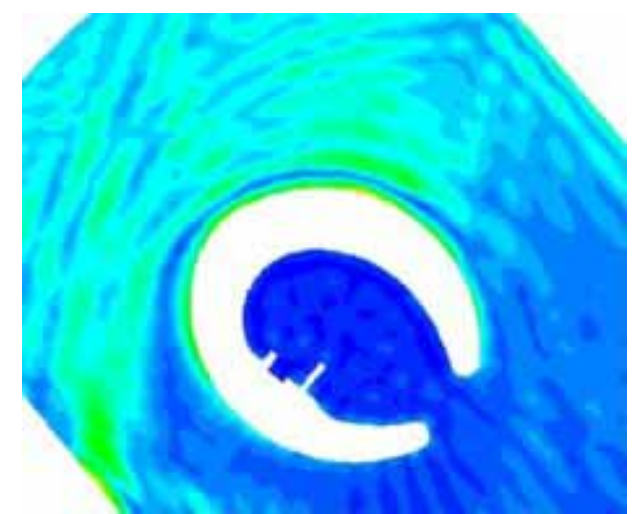

Figure 5. Projet- houle annuelle de NW Tp : $12 \mathrm{~s}$.

\section{Prédimensionnement des GSC}

L'étude de la stabilité des tubes en géotextile est complexe. En effet, leur comportement est conditionné notamment par :

- La profondeur d'eau et les conditions de houles auxquelles ils sont soumis ;

- La non-rigidité de la structure ;

- Les dimensions de la structure et le taux de remplissage ;

- Le matériau de remplissage ;

- Les caractéristiques mécaniques du géotextile (...).

Dans la littérature, il n'existe pas, à notre connaissance, de formulations permettant d'intégrer l'ensemble de ces paramètres. Cependant, plusieurs publications permettent d'estimer la stabilité d'un tube/container géotextile en fonction de ses dimensions et des caractéristiques de houle ou de courant auxquels il est soumis. Deux procédures sont mises en œuvre :

- A partir du degré de remplissage souhaité et de la taille du tube vide, on déterminera les caractéristiques géométriques du tube rempli et une première approximation des 


\section{Thème 2 - Dynamique sédimentaire}

conditions hydrodynamiques maximales permises d'après BEZUIJEN et VASTENBURG (2012), (voir figure 6) ;

- A partir des dimensions définies ci-dessus, on appliquera des critères de stabilité pour s'assurer, selon différentes formulations que le tube sera stable en fonction des conditions hydrodynamiques auxquelles il sera soumis.

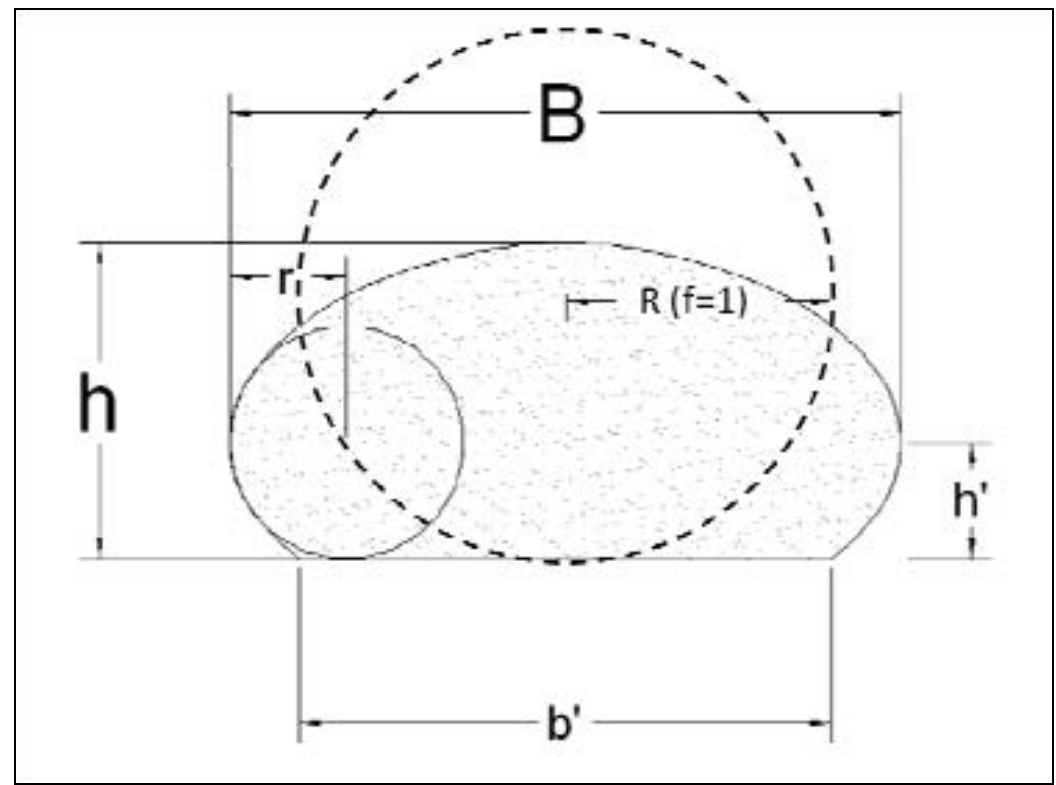

Figure 6. Dimensions du tube en géotextile pour un degré de remplissage f.

D'après BEZUIJEN et VASTENBURG (2012), on peut déterminer les caractéristiques du tube mis en œuvre à partir des dimensions connues du géotextile (ici $\mathrm{D}=2 \mathrm{R}=4,5 \mathrm{~m}$ ) et du degré de remplissage.

$$
\begin{aligned}
& h \geq(1-\sqrt{1-f}) \cdot D \\
& B \leq h+\frac{1}{2} \cdot \pi \cdot(D-h)
\end{aligned}
$$

Tableau 2. Dimensions estimées du tube en fonction du degré de remplissage.

\begin{tabular}{lllll}
\hline$f[-]$ & $h[\mathrm{~m}]$ & $B[-]$ & $S[\mathrm{~m}]$ & $A\left[\mathrm{~m}^{2}\right]$ \\
\hline 1 & 4.50 & 4.50 & 14.14 & 15.90 \\
0.9 & 3.20 & 5.40 & 14.33 & 14.54 \\
0.8 & 2.63 & 5.76 & 14.06 & 12.64 \\
0.7 & 2.21 & 6.05 & 14.33 & 11.33 \\
0.6 & 1.82 & 6.28 & 13.74 & 9.30 \\
\hline
\end{tabular}

En première approche, il est aussi possible d'estimer (Eq 3) les valeurs critiques des hauteurs de houles ou courants (uniformes) en dessous desquelles un tube en géotextile de dimensions connues sera stable : 


\section{XIII ${ }^{\text {èmes }}$ Journées Nationales Génie Côtier - Génie Civil \\ Dunkerque, 2-4 juillet 2014}

$$
\begin{aligned}
& \frac{H_{s}}{\Delta_{t} \cdot h} \leq 1 \\
& \frac{u_{c r}}{\sqrt{g \cdot \Delta_{t} \cdot h}} \leq\left\{\begin{array}{cc}
1 & \text { SCHIERECK }(2004) \\
0,5 \text { à } 1 & \text { PILARCZYK(2000) }
\end{array}\right.
\end{aligned}
$$

En considérant un degré de remplissage de 0,8 , on estime la valeur critique de hauteur de houle admissible est $H_{s}<2,4 \mathrm{~m}$ et la vitesse critique de courant à : $u_{c r}<2,4 \mathrm{~m} / \mathrm{s}$.

Le prochain objectif est de pouvoir déterminer la largeur critique de l'ouvrage pour une hauteur et une longueur donnée. Un bilan des forces appliquées aux tubes est réalisé, à l'image de la méthode utilisée par RECIO \& OUMERACI (2007) sur le comportement de "Geotextile Sand Containers". Dans cette analyse les effets de déformation des tubes sur la stabilité hydrodynamique de ceux-ci ne sont pas pris en compte. Ces forces sont listées ci-après :

- Force de traînée : $F_{D}=0.5 \rho_{w} u^{2} C_{D} A_{S}$

- Force inertielle : $F_{M}=\rho_{w} C_{M} V \frac{\partial u}{\partial t}$

- Force de soulèvement : $F_{L}=0.5 \rho_{w} u^{2} C_{L} A_{T}$

- Poids relatif GST (Geotextile Sand Tube) : $F_{G S T}=\left(\rho_{s}-\rho_{w}\right) u^{2} g V$

Ces forces dépendent donc de la vitesse, de l'accélération des particules fluides, des dimensions du GST et de coefficients traduisant l'emprise des structures sur l'écoulement. Ils sont fonction du régime de l'écoulement mais aussi de la géométrie de la structure. La principale difficulté pour l'estimation des longueurs critiques de stabilité réside dans l'estimation de ces coefficients. S'ils sont assez bien connus dans le cadre de piles (MORISON et al., 1953) car très utilisés en offshore, ils sont peu renseignés dans le cadre de tubes en géotextile. Les travaux de RECIO \& OUMERACI (2007) permettent d'estimer ces coefficients pour des configurations diverses d'empilements des GSC. Le calcul est réalisé pour un empilement de trois GSC (deux tubes joints posés sur le terrain naturel et un au-dessus). Les résultats sont issus des tests réalisés en canal avec un nombre de Reynolds inférieur à $10^{6}$. La longueur critique du GSC est calculée de deux manières, considérant la longueur critique de glissement et la longueur critique de renversement. La valeur maximale de ces longueurs sera retenue comme le minimum de dimensionnement. Les formules de calcul utilisées pour déterminer la largeur critique de dimensionnement ont été adaptées à partir des travaux de RECIO \& OUMERACI (2007) pour correspondre au projet. Pour cela, nous considèrerons une hauteur de 2,7 m et une largeur de $5.7 \mathrm{~m}$. Les formulations deviennent : 


$$
\begin{aligned}
& l_{c(\text { sliding })} \geq u^{2} \frac{C_{D}+\mu \cdot 2,5 C_{L}}{\mu \Delta g-C_{M} \partial u / \partial t} \\
& l_{c(\text { overturning })} \geq u^{2} \frac{0,1 C_{D}+1,25 C_{L}}{0,5 \Delta g-0,1 C_{M} \partial u / \partial t}
\end{aligned}
$$

Avec $\mu$ le coefficient de frottement, et $\Delta=\frac{\rho_{s}-\rho_{w}}{\rho_{w}}$.

Les conditions de houle retenues pour le calcul sont reportées dans le tableau 3. Il s'agit de considérer les niveaux d'eau extrêmes considérés en fonction de la position sur l'île. Pour les deux cas, il s'agit de vérifier la stabilité des GST en fonction de leurs dimensions et en réponse à des houles susceptibles de se produire dans le secteur.

\begin{tabular}{|c|c|c|c|c|c|c|c|}
\hline \multirow{2}{*}{ Cas } & \multicolumn{2}{|c|}{ Caractéristiques GSC } & \multicolumn{5}{|c|}{ Houle } \\
\hline & $\boldsymbol{h}$ & $B[m]$ & $T[s]$ & $H[m]$ & $L_{0}[m]$ & $L[m]$ & $d[m]$ \\
\hline 1 & 2.7 & 5.7 & 14 & 1 & 306 & 126 & $6.5+2.25$ \\
\hline 2 & 2.7 & 5.7 & 15 & 1 & 351 & 136 & $6.5+2.35$ \\
\hline
\end{tabular}

Tableau 3. Conditions de houles sélectionnées pour le calcul de $l_{c}$

Les résultats sont présentés dans le tableau 4. Les longueurs critiques sont divisées par deux pour obtenir la largeur critique du GSC. Sont donnés à titre indicatif les résultats de vitesse et d'accélération calculés à mi-hauteur du GSC $(1,2 \mathrm{~m})$.

Tableau 4. Résultats de largeur minimale de GST.

\begin{tabular}{lllll}
\hline Cas & $\boldsymbol{I}_{\boldsymbol{c}}$ (sliding)/2 (m) & $\boldsymbol{I}_{\boldsymbol{c}}$ (overturning)/2 $(\boldsymbol{m})$ & $\boldsymbol{u}(\boldsymbol{m} / \mathbf{s})$ & $\boldsymbol{a}\left(\mathbf{m} / \mathbf{s}^{2}\right)$ \\
\hline 1 & 0.21 & 0.11 & 0.64 & 0.29 \\
2 & 0.20 & 0.11 & 0.63 & 0.26 \\
\hline
\end{tabular}

Les dimensions initialement prévues en termes de longueur et de largeur répondent donc aux critères de stabilité dans le cas d'une structure rigide.

A titre de comparaison, on peut citer quatre autres critères de stabilité de structures composées de GSC présents dans la littérature dont les configurations géométriques sont proches du projet :

- BOUYZE \& SHRAM (1990), pour le calcul d'un critère de stabilité sous l'action d'un courant uniforme ;

- PILARCZYK (2000) : pour le calcul d'un critère de stabilité des tubes en géotextile ;

- HINZ et al. (2002) : pour l'estimation de la largeur caractéristique critique de GSC dans le cas d'une structure de type digue infranchissable ;

- GRÜNE et al. (2006) : pour l'estimation de la largeur caractéristique critique de GSC. 


\section{XIII ${ }^{\text {èmes }}$ Journées Nationales Génie Côtier - Génie Civil \\ Dunkerque, 2-4 juillet 2014}

Tableau 5. Largeur critique des géotubes $(m)$ - Comparaison de formules de stabilité

\begin{tabular}{|c|c|c|c|c|}
\hline Cas & $\begin{array}{l}U_{c r} /(\boldsymbol{g} \Delta \boldsymbol{D})^{0.5} \sim \mathbf{0 , 5 - 1} \\
\text { BOUYZE \& SHRAM (1990) }\end{array}$ & $\begin{array}{l}\boldsymbol{b}=(\mathbf{1 . 1}-\mathbf{1 . 2}) \boldsymbol{H}_{\boldsymbol{B}}<\mathbf{1} \\
\text { PILARCZYK }(2000)\end{array}$ & $\begin{array}{l}\mathbf{I}=\boldsymbol{H}_{\boldsymbol{s}}^{3 / 4} \boldsymbol{T}^{\mathbf{1 / 2}} / \mathbf{1 . 7 4} \\
\left(\rho_{G S C} / \boldsymbol{\rho}_{w} \mathbf{- 1}\right) \\
\text { HINZ et al. }(2002)\end{array}$ & $I=\left(10 V \rho_{s}\right)^{1 / 3}$ \\
\hline 1 & 0.11 & 0.30 & 3.1 & 4.8 \\
\hline 2 & 0.11 & 0.30 & 3.2 & 4,8 \\
\hline
\end{tabular}

Les résultats des différents critères de stabilité sont donnés dans le tableau 4. Il apparaît que l'ensemble des formulations concluent à la stabilité des GSC pour les 2 cas proposés.

Les caractéristiques du géotextile doivent être adaptées par rapport à la résistance de la membrane au matériau de remplissage et à sa perméabilité. Contrairement aux géotextiles non tissés, les géotextiles tissés ont une haute résistance à la traction $\left(40<T_{m}<300 \mathrm{kN} / \mathrm{m}\right)$ avec un faible niveau de déformation maximale admissible $\left(1 \%<\varepsilon_{m}<15 \%\right)$. La perméabilité ainsi que la dimension de l'ouverture de filtration du géotextile sont deux caractéristiques déterminantes dans le choix du géotextile. La granulométrie des matériaux de remplissage a été adaptée au GSC tissé sélectionné $\left(O_{90}=425 \mu m\right)$.

\section{La digue de protection}

S'agissant de la coupe de projet pour la digue de protection en géotextile, une variante est proposée pour le profil intérieur :

- Une base solide avec 3 tubes en géotextile dont l'arase sera située approximativement au zéro cote marine ;

- Une zone de plage de $20-25 \mathrm{~m}$;

- Un 4ème tube en géotextile dans la partie supérieure (hors d'eau) qui assure la stabilité du talus arrière à la cote supérieure souhaitée.

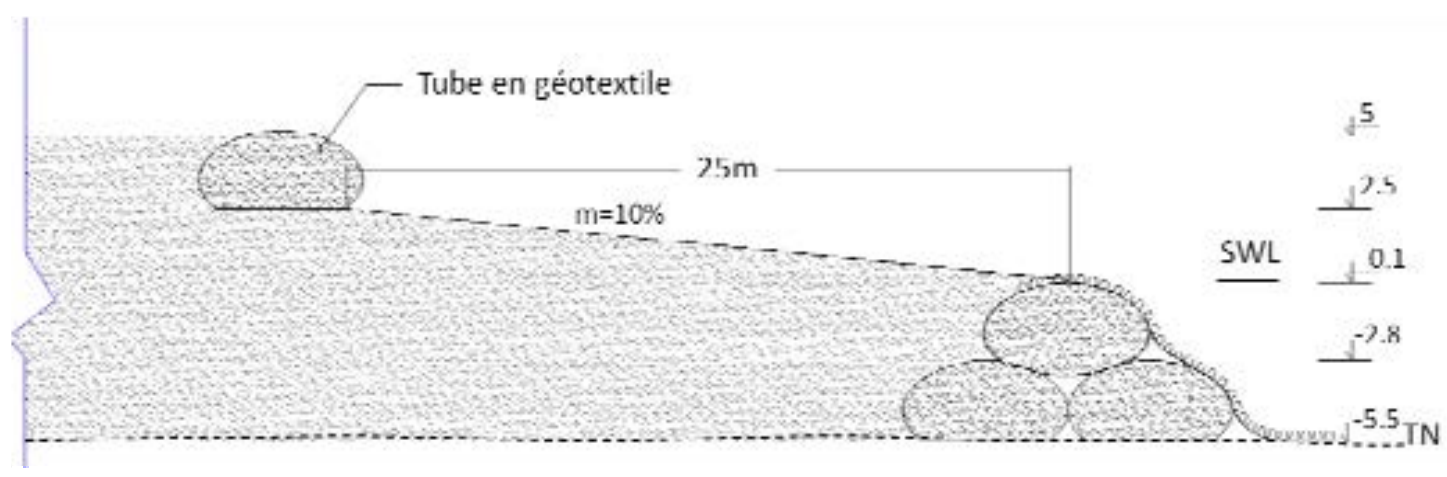

Figure 7. Profil proposé pour la partie intérieure.

Le profil extérieur retenu pour le projet est représenté sur la figure 8. Considérant l'angle du talus constitué d'empilements de tubes, nous conseillons de conserver un 


\section{Thème 2 - Dynamique sédimentaire}

angle de $30^{\circ}$ correspondant à l'angle de frottement du sédiment de remplissage. Afin de limiter l'usage prématuré des tubes en géotextile face à l'exposition aux UV ou à l'usure générée par les conditions de houle un tapis géotextile supplémentaire (FlexMat - voir figure 8), sur lequel sont fixés des blocs de béton, recouvre la structure.

Le terrain naturel atteint une cote variable entre -3.5 à $-6.5 \mathrm{mCM}$. Il convient de déterminer la cote d'arase de l'ouvrage en fonction des niveaux d'eau et de l'agitation :

- Le niveau d'eau à pleine mer VE : +2.35 mCM ;

- La surcote générée par des conditions de tempête : $+0.5 \mathrm{~m}$;

- La surélévation du niveau marin due aux changements climatiques : $+0.1 \mathrm{~m}$;

- Les calculs de run-up réalisés montrent que celui-ci ne dépasse pas $4 \mathrm{~m}$ pour une houle centennale : $R u 2 \%<3.8 \mathrm{~m}$.

Au vu de l'ensemble de ces éléments, il convient de retenir une valeur $z_{a}$ pour la côte d'arase de l'ouvrage supérieure ou égale à $+6,75$ mCM.

Considérant ces résultats et les hauteurs d'eau atteintes définies dans la section précédente, nous proposons le découpage suivant en fonction de deux zones géographiques avec les cotes d'arase de l'ouvrage en fonction des houles de différentes périodes de retour (voir figure 9).

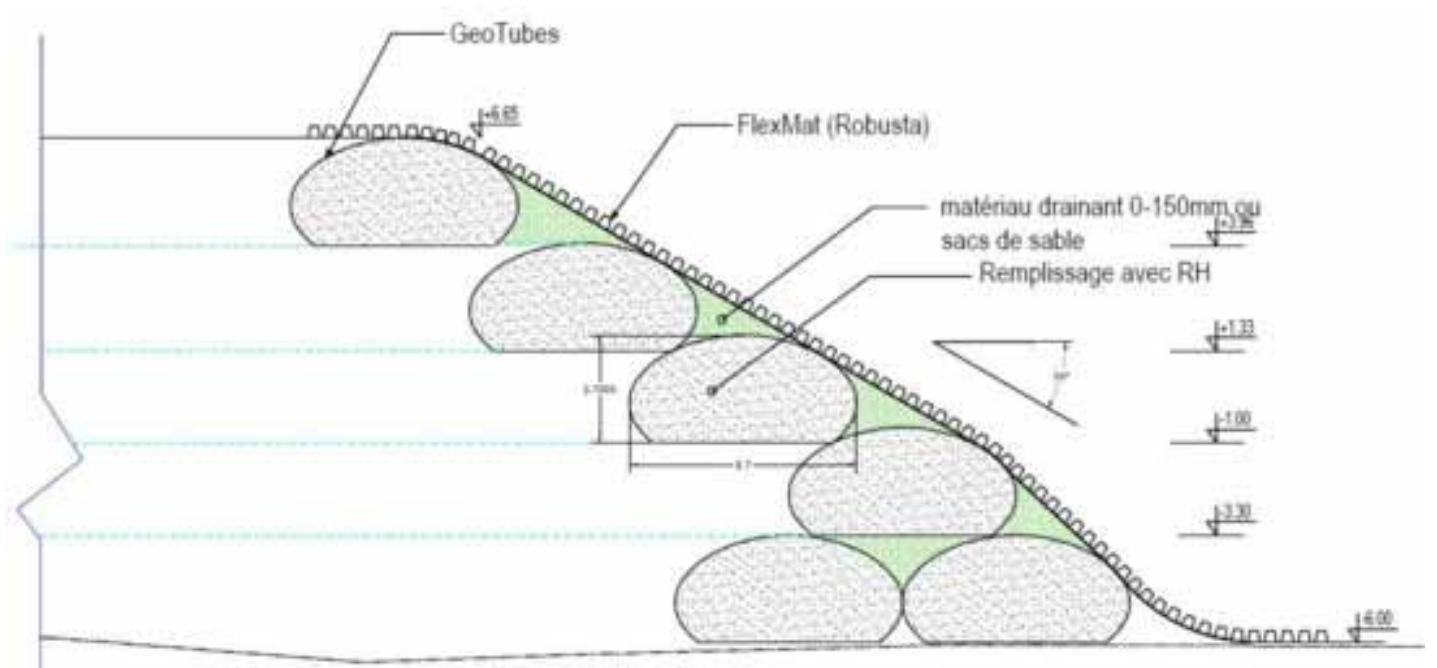

Figure 8. Schéma de principe pour un niveau de terrain naturel à -6.5 mCM et un niveau d'eau de période de retour de 50 ans. 


\section{XIII ${ }^{\text {èmes }}$ Journées Nationales Génie Côtier - Génie Civil \\ Dunkerque, 2-4 juillet 2014}

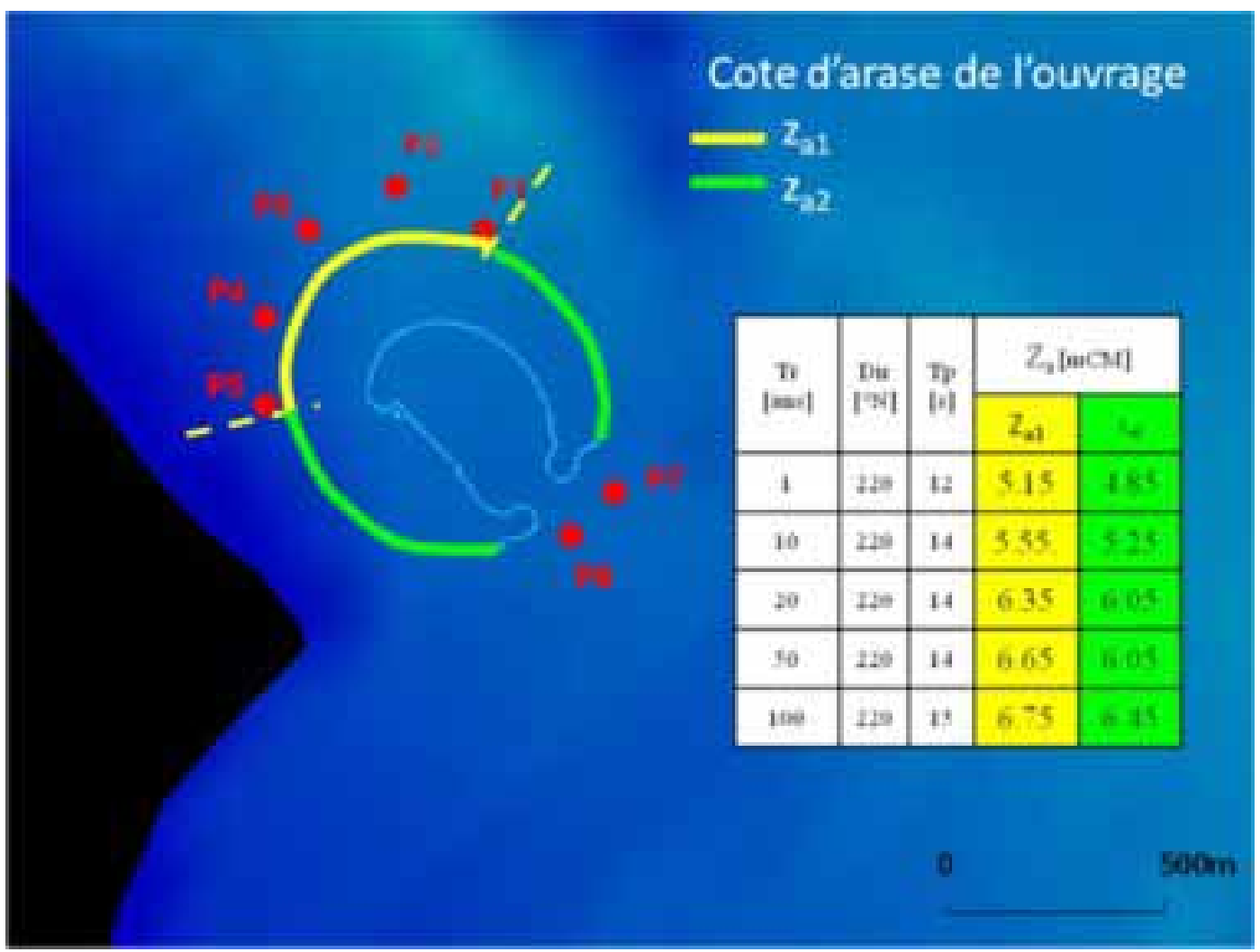

Figure 9. Cotes d'arase minimale des ouvrages en fonction de Tr.

\section{Conclusion}

Pour un degré de remplissage à $80 \%$, une hauteur du tube à $2.6 \mathrm{~m}$ et une largeur de $5,8 \mathrm{~m}$, les tubes sont stables au regard des contraintes hydrodynamiques ( $\mathrm{Hs}<2 \mathrm{~m}$ ) et pour une vitesse critique de courant de $2.4 \mathrm{~m} / \mathrm{s}$. La granulométrie du matériau de remplissage $\left(d_{50} \# 400 \mu \mathrm{m}\right)$ et le mélange eau/sédiments du remplissage à $20 \%$ sont conseillés. Deux profils de digue sont proposés : un pour la partie extérieure et un pour l'intérieur de l'îlot. Pour la partie extérieure, la cote d'arase est fixée à +6,75 mCM correspondant à la période de retour centenale. C'est la cote de projet avec une pente de $30^{\circ}$ et à la base un assemblage de 3 tubes en pyramide. Pour la partie intérieure, moins exposée à l'agitation une plage de sable est proposée pour amortir l'effet du clapot et valoriser l'espace de la zone touristique. Ce concept d'îlot de sable qui s'est développé dans les émirats peut aussi s'envisager dans d'autres environnements (tropicaux ou équatoriaux) à condition d'être suffisamment protégé des agents hydrodynamiques, de disposer de réserves de sables de bonne qualités et de profondeurs suffisamment faibles.

\section{Références bibliographiques}

BEZUIJEN A., VASTENBURG E.W. (2012). Geosystems: design rules and applications. Deltares, Delft, the Netherlands, edited by CRC Press, 164 p. http://dx.doi.org/10.1201/b13738 
BOUYZE, SHRAM (1990): Stabiliteit van Grondkribben en Onderwatergolbrekers Opgebouwd uit Zandworsten. TU-Delft, Studentarbeit (Master Student Project Report). GRÜNE J., SPARBOOM U., SCHMIDT-KOPPENHAGEN R., WANG Z., OUMERACI H. (2006). Stability tests of geotextile sand containers for monopile scour protection. Proceedings 30th International Conference Coastal Engineering (ICCE), Vol. 5, San Diego, California, USA, pp 5093-5105.

HINZ M., BLECK M., OUMERACI H. (2002) Großmaßstabliche Untersuchungen zur Hydraulischen Stabilität geotextiler Sandcontainer unter Wellenbelastun. LWI-Report no. 878. Leichtweiss Intitute, Germany.

MORISON J.R., JOHNSON J.W., O'BRIEN M.P. (1953). Experimental studies of forces on piles. Coast. Eng. Proc. 1(4), pp 340-370.

PILARCZYK (2000): Geosynthetics and geosystems in hydraulic and coastal engineering. A.A. Balkema, Rotterdam, the Netherlands.

RECIO J., OUMERACI H. (2007). Effect of deformations on the hydraulic stability of coastal structures made of geotextile sand containers. Journal of Geotextiles and Geomembranes, Vol. 25, pp 278-292. http://dx.doi.org/10.1016/j.geotexmem.2007.02.006

SCHIERECK (2004). Introduction to bed, bank and shore protection. DUP Blue Print, Delft. 